\title{
Frequency dependency of the relationship between perceived auditory source width and the interaural cross-correlation coefficient for time-invariant stimuli
}

\author{
Russell Mason, a) Tim Brookes, and Francis Rumsey \\ Institute of Sound Recording, University of Surrey, Guildford, Surrey, GU2 7XH, England
}

(Received 27 November 2003; revised 2 December 2004; accepted 6 December 2004)

\begin{abstract}
Previous research has indicated that the relationship between the interaural cross-correlation coefficient (IACC) of a narrow-band sound and its perceived auditory source width is dependent on its frequency. However, this dependency has not been investigated in sufficient detail for researchers to be able to properly model it in order to produce a perceptually relevant IACC-based model of auditory source width. A series of experiments has therefore been conducted to investigate this frequency dependency in a controlled manner, and to derive an appropriate model. Three main factors were discovered in the course of these experiments. First, the nature of the frequency dependency of the perceived auditory source width of stimuli with an IACC of 1 was determined, and an appropriate mathematical model was derived. Second, the loss of perceived temporal detail at high frequencies, caused by the breakdown of phase locking in the ear, was found to be relevant, and the model was modified accordingly using rectification and a low-pass filter. Finally, it was found that there was a further frequency dependency at low frequencies, and a method for modeling this was derived. The final model was shown to predict the experimental data well. (C) 2005 Acoustical Society of America. [DOI: 10.1121/1.1853113]
\end{abstract}

PACS numbers: 43.66.Pn, 43.66.Rq, 43.66.Ba, 43.55.Mc [AK] Pages: 1337-1350

\section{INTRODUCTION}

It has been shown that the results of measurements based on the interaural cross-correlation coefficient (IACC) can be used to predict a number of aspects of binaural perception. Whereas recent research has suggested that it is not an accurate representation of the physiology of the binaural hearing process (Brand et al., 2002), the predictions of models of binaural perception based on the IACC have shown remarkable similarity to experimental data relating to lateralization (e.g., Sayers, 1964; Okano, 2000; Constan and Hartmann, 2001), binaural detection thresholds (e.g., Sayers and Cherry, 1957; Robinson and Jeffress, 1963; Bernstein and Trahiotis, 1992), and the perceived source width of an auditory stimulus (e.g., Chernyak and Dubrovsky, 1968; Plenge, 1972; Kurozumi and Ohgushi, 1983; Blauert and Lindemann, 1986).

The relationship between the IACC and binaural detection thresholds has been researched in detail, and the majority of relevant fundamental factors such as frequency dependency (e.g., Bernstein and Trahiotis, 1996b), and temporal resolution (e.g., Culling and Summerfield, 1998), have been investigated in a controlled manner. The relationship between the IACC and perceived auditory source width, however, has not been investigated in as much detail. The aim of this paper is to investigate the frequency dependency of the relationship between the IACC of an auditory stimulus and the perceived auditory source width of that stimulus, and to model this dependency such that it can be incorporated in an auditory source width measure.

In the late 1960s, Chernyak and Dubrovsky (1968)

a)Electronic mail: r.mason@surrey.ac.uk showed that there was a negative relationship between the IACC and the perceived auditory source width of noise stimuli that were presented over headphones. This relationship was also found by Keet (1968), who used musical signals that were reproduced over loudspeakers. This inverse relationship between the IACC and the perceived auditory source width has been confirmed by other investigations using a range of stimuli (Kurozumi and Ohgushi, 1983; Morimoto et al., 1993). From this, a number of researchers have developed models, based on the IACC, to predict the perceived source width of auditory stimuli for sound reproduction (e.g., Sakai et al., 1980; Tohyama and Suzuki, 1989) and auditorium acoustics (e.g., Ando, 1985; Morimoto and Iida, 1995; Hidaka et al., 1995). This research has resulted in models that match perception reasonably well, and has led to the standardization of some methods (ISO 3382, 1997). However, in spite of the growing popularity of these models, there are arguments against their practicality (e.g., Griesinger, 1997; de Vries et al., 2001), and there are also a number of fundamental factors that have not yet been fully addressed in a controlled manner.

One such factor is the effect of audio frequency on the relationship between the IACC and the perceived source width of an auditory stimulus. Investigations by a number of researchers have indicated the existence of this effect (e.g., Perrott et al., 1980; Blauert and Lindemann, 1986; Yanagawa et al., 1990), but methods to model it have not yet been derived.

The experiments that are reported in this paper investigate the frequency dependency of the relationship between the IACC and perceived auditory source width in detail, with the aim to identify and model the separate contributory components, in order to develop an objective auditory source 


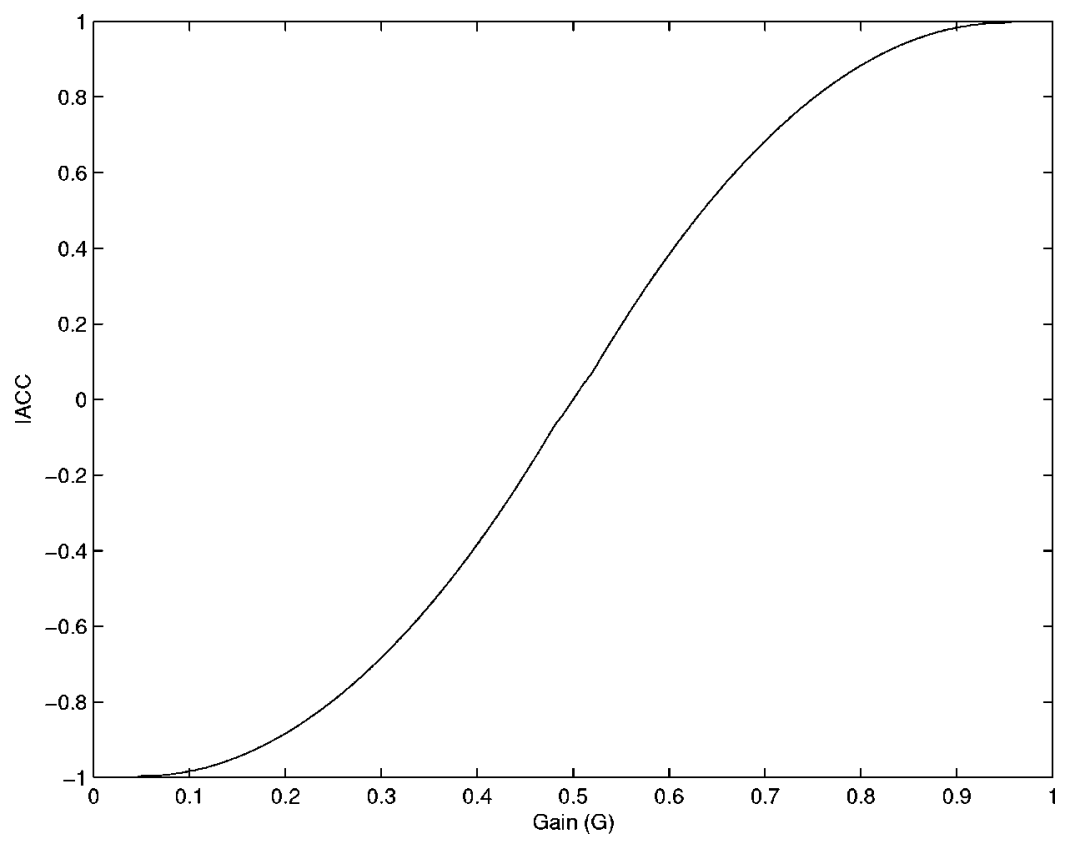

FIG. 1. Relationship between the gain value, $G$, and the resulting IACC for stimuli created using the method described in Eqs. (1)-(4).

width measure that is suitable for a wide range of stimulus types. Additionally, it is expected that the research reported in this paper will lead to the inclusion of an IACC-based auditory source width model in a more complete binaural hearing model, similar to that proposed by Morimoto et al. (1995). The experiments reported herein all employ continuous narrow-band stimuli, and it is acknowledged that further work may be required to adjust the resulting model for use with stimuli that are more externally valid. Detailed information on the IACC-based calculations used in this paper is contained in the Appendix.

\section{METHOD}

\section{A. Stimuli}

In order to undertake the experiment in a controlled manner and limit extraneous variables as much as possible, stimuli with specific characteristics were required. A detailed discussion of the requirements of the stimuli for the experiments that are described in this paper is provided by Mason et al. (2003); the pertinent information is summarized here. First, testing of frequency dependence requires stimuli with a narrow bandwidth - ideally within a single critical band, i.e., approximately $35 \mathrm{~Hz}$ at low frequencies and widening with increasing center frequency (Glasberg and Moore, 1990). Second, accurate mapping of a perceived effect to a given measured value requires stimuli that are time invariant in terms of that metric. As any measurement of time invariance is dependent on the temporal resolution of that measurement, the optimum integration window needs to be determined. Previous studies into the perception of temporal variations in IACC have suggested that this can be modeled by a time constant of between 35 and $243 \mathrm{~ms}$, depending on the experiment setup and the individual subject (Grantham and Wightman, 1979; Boehnke et al., 2002). For these experiments the shortest integration time (therefore the most critical value resulting from the most sensitive subjects) from previous studies was used, namely $35 \mathrm{~ms}$. Finally, to be able to examine the effect of frequency dependency at a wide range of values of IACC requires stimuli whose IACC can be varied in small steps across the entire range of values while minimizing any other perceivable variations.

Previous experiments that investigated relationships between the IACC and perceived auditory source width used a wide range of stimuli, including music (e.g., Keet, 1968; Morimoto and Iida, 1995) and noise (e.g., Chernyak and Dubrovsky, 1968; Blauert and Lindemann, 1986). The main problem with these types of stimuli is that the IACC can vary a great deal over time, as shown by Mason et al. (2003), which makes it difficult to specify the actual IACC value to which a given perceptual effect relates, be it the maximum, minimum, or some type of average.

The stimuli chosen for use in the experiments described in this paper are based on suppressed carrier amplitude modulation with a time offset between the channels. Two signals, $s_{1}$ and $s_{2}$, are created as shown in Eqs. (1) and (2); where $f_{c}$ is the carrier frequency, $f_{m}$ is the modulation frequency, and $t_{m}$ is the time offset defined as $\left[1 /\left(4 f_{m}\right)\right]$

$$
\begin{aligned}
& s_{1}=\sin \left(2 \pi f_{c} t\right) \sin \left(2 \pi f_{m} t\right), \\
& s_{2}=\sin \left(2 \pi f_{c}\left[t+t_{m}\right]\right) \sin \left(2 \pi f_{m}\left[t+t_{m}\right]\right) .
\end{aligned}
$$

A two-channel output signal, $l$ and $r$, is then created by mixing signals $s_{1}$ and $s_{2}$; one in phase and one phase inverted between the channels, as shown in Eqs. (3) and (4). Assuming that the stimuli are reproduced to the subjects over headphones, the IACC of the output signal can be altered by varying the gain value $G$. A range of values of $G$ of 0 to 1 results in a range of values of IACC of -1 to 1 , and it is possible to set the IACC to be any value within this range:

$$
\begin{aligned}
& l=G s_{1}+(1-G) s_{2}, \\
& r=G s_{1}-(1-G) s_{2} .
\end{aligned}
$$

The relationship between the gain $G$ and the resulting IACC is independent of $f_{c}$ for the range used, but is not linear, as is shown in Fig. 1. This means that a linear variation in $G$ 
results in a small variation in the resulting IACC close to 1 and -1 where the ear is more sensitive to small changes, and a larger variation in the resulting IACC close to 0 where the ear is less sensitive to small changes (Gabriel and Colburn, 1981; Boehnke et al., 2002).

The modulation frequency $-f_{m}$ in Eqs. (1) and (2) —of this type of stimulus affects the bandwidth and the time invariance of the IACC in a contrary manner, which requires a compromise. For the experiments described in this paper, the value of $f_{m}$ was set to be $20 \mathrm{~Hz}$, which resulted in a bandwidth of $40 \mathrm{~Hz}$ (slightly wider than a critical band at low center frequencies) and a constant IACC over time for an integration window of $50 \mathrm{~ms}$ or longer (resulting in an IACC that is perceptually time invariant for the majority of listeners, excluding the most sensitive).

The center frequency of a stimulus is set by altering the carrier frequency, $f_{c}$ in Eqs. (1) and (2). It was confirmed that the IACC of the stimuli could be adjusted by altering the value of $G$ in Eqs. (3) and (4), and that this caused only minimal changes in the perceived timbre of the stimuli. The interested reader is referred to Mason et al. (2003) for more information.

The stimuli were generated using computer software, which allowed variation of $G$ in real time in 128 equal steps between 0.5 and 1 (giving a range of IACC of 0 to 1 as shown in Fig. 1). The output signal was fed through a 16-bit DAC to a pair of Sennheiser HD-580 headphones.

The stimuli were reproduced at a sound pressure level (measured individually at each ear) corresponding to 70 phons, as derived from the equal loudness contours contained in ISO 226 (1987). Whereas the data contained in ISO 226 are intended for pure tone stimuli as opposed to these two-tone experiment stimuli, informal listening by the authors confirmed that the stimuli were all of similar loudness after alignment.

\section{B. Procedure}

The experiments were conducted using a "method of adjustment" paradigm (Gescheider, 1997), where the subjects were asked to adjust a variable stimulus to be the same perceived auditory source width as a given fixed stimulus. The fixed stimuli were presented individually, and were continuously looped so that the subjects could take as long as required to adjust the auditory source width of the variable stimulus. The subjects could switch between the variable and fixed stimuli whenever and as often as they required, and could move on to the next fixed stimulus when they were satisfied with their result. The switching between the stimuli was instantaneous, with a 20 -ms cross-fade to avoid audible clicks. The user interface was configured so that the subjects could alter the IACC of the variable stimulus either by moving an on-screen slider with a mouse or by using a hardware slider. The subjects could switch between the stimuli using either the mouse or hardware buttons. The order of presentation of the stimuli was randomized by the computer to avoid biases caused by order effects; this also rendered the test double blind (i.e., neither the subjects nor the experimenter knew which fixed stimulus was which).

The benefits of the method of adjustment procedure over other experimental methods are that it is relatively efficient in terms of the amount of time required to undertake the experiment, and the intrasubject repeatability is high (Hesse, 1986). In addition, as the subjects have an active role in the experiment, it is less likely that their performance will suffer due to boredom or fatigue (Cardozo, 1965). However, there are two main drawbacks to the method of adjustment procedure. First, it is possible that in an experiment with a large number of repetitions that require similar adjustments to be made in each case, a bias will be caused by habituation and expectation (Gescheider, 1997). Second, the method lacks the robustness of other procedures, i.e., it is dependent on the judgment of the subjects that a match is "good enough" (Wier et al., 1976), as opposed to a strict statistical method such as that used in a two-alternative forced choice (2AFC) experiment (Levitt, 1971). It is apparent that the effects of these drawbacks can be reduced by the use of carefully selected subjects and, in view of this, it was decided that the advantages of the procedure outweighed the disadvantages for these experiments.

\section{Subjects}

As the method of adjustment procedure is strongly dependent on the ability of the subject, a pilot experiment was used to select a small panel of suitable listeners. This used the same procedure and a subset of the stimuli from the main experiments, and also served to train the subjects in the experiment task and to enable them to become familiar with the types of stimuli that were used throughout. The subjects were selected based on their consistency (between judgments for multiple iterations of the same stimuli) and accuracy (of matching the IACC of a fixed stimulus that had the same center frequency as the variable stimulus). Seven subjects were selected from the 25 who undertook the pilot experiment. The selected subjects included one female and six males, with an age range of 21 to 35 , one of which was the first author. They were either final year undergraduates, postgraduates, or staff from the Institute of Sound Recording, and could therefore be considered to be experienced critical listeners. The subjects were instructed that there were no correct answers in the experiment, and that they would only be rated on the consistency of their judgments.

\section{EXPERIMENT 1-EFFECT OF FREQUENCY ON PERCEIVED AUDITORY SOURCE WIDTH WHEN IACC $=1$}

\section{A. Introduction}

The research of Perrott et al. (1980) showed that the perceived auditory source width (which they termed "tonal volume") of an auditory stimulus was dependent on the center frequency, even for a simple diotic stimulus (i.e., a signal that is identical at each ear and therefore has an IACC of 1), such as a single sine tone. As it is known that the IACC has an inverse relationship with the perceived auditory source width, it can be assumed that stimuli with an IACC of 1 will represent the narrowest condition at a particular frequency. In view of this, the first experiment examined the effect of the frequency of a stimulus on its perceived auditory source 


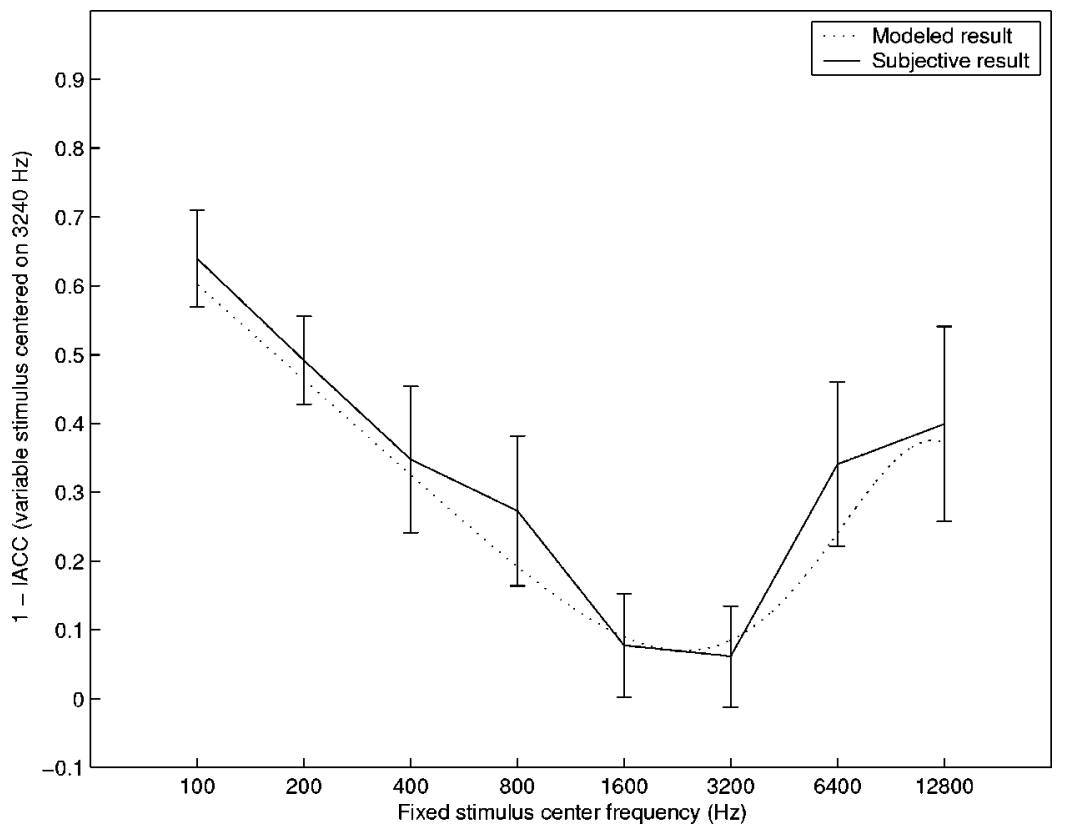

FIG. 2. Means and associated $95 \%$ confidence intervals of the 1-IACC of the variable stimulus centered on $3240 \mathrm{~Hz}$ that the subjects judged to have the same auditory source width as each fixed stimulus in experiment 1 (subjective result), together with the results predicted by Eq. (5) (modeled result).

width for an IACC of 1 , in order to determine a baseline from which any changes in the perceived auditory source width caused by variations in the IACC can be calculated.

\section{B. Stimuli}

Stimuli based on amplitude modulation, as described above, were used in the experiment. The stimuli were created using Eqs. (1) $-(4)$, with $f_{m}=20 \mathrm{~Hz}$ and $G=1.0$. In order to examine the effect of the frequency of the stimulus on the perceived auditory source width, fixed stimuli with a range of values of $f_{c}$ were used with octave spacing: 100, 200, 400, 800, 1600, 3200, 6400, and $12800 \mathrm{~Hz}$.

The variable stimulus that was used in the experiment was created using $f_{m}=20 \mathrm{~Hz}$ and $f_{c}=3240 \mathrm{~Hz}$. The value of $f_{m}$ was chosen to match the fixed stimuli, and the value of $f_{c}$ was chosen (from informal listening by the experimenters) to give a range of variation of auditory source width for the variable stimulus that encompassed the range of widths present in the fixed stimuli.

\section{Procedure}

There were eight fixed stimuli in the experiment; for each of these the subjects were asked to adjust the variable stimulus until they judged it to have the same auditory source width as the fixed stimulus. The experiment comprised four repetitions, each presented in a different random order. All seven selected subjects undertook the full experiment.

\section{Results}

The results of the subjective experiment were analyzed by calculating the IACC of the variable stimulus that the subjects judged to have the same perceived auditory source width as each fixed stimulus. The means and associated $95 \%$ confidence intervals of these results are shown as the subjective result in Fig. 2, with the center frequency of each fixed stimulus along the $x$ axis and the IACC value subtracted from 1 (1-IACC) of the matched variable stimulus on the $y$ axis. The IACC scale is inverted in this manner to give a positive correlation with the perceived auditory source width, as discussed earlier. The wide variation in 1-IACC, resulting from changes in the center frequency of the fixed stimulus, equates to a wide variation in perceived auditory source width, despite the fact that the IACC was set to be 1 for all the fixed stimuli. It may be interpreted from these results that the low-frequency fixed stimuli were perceived to be widest, with a monotonic decrease in the auditory source width to approximately $1600 \mathrm{~Hz}$. Above $3200 \mathrm{~Hz}$ the perceived auditory source width appears to rise again.

The results from this experiment can be modeled by a frequency-dependent equation, as shown in Eq. (5), where $b$ predicts the 1-IACC of the variable stimulus whose auditory source width would be judged to match that of a fixed stimulus with center frequency $f$. This is shown in Fig. 2 as the modeled result:

$b=\frac{\sin [(5 \sqrt{f}+4000) / 100]+1}{f^{0.2}}+0.07, \quad 100 \leqslant f \leqslant 12800$.

It is reasonable to expect that the perceived widths of two similar stimuli having similar center frequencies and the same IACC and loudness will be approximately equal. Therefore, when the center frequency of the fixed stimulus is close to that of the variable stimulus, it is reasonable to expect that the subjects will adjust the IACC of the variable stimulus to be similar to the IACC of the fixed stimulus. Based on this expectation, the 1-IACC of the variable stimulus adjusted to match the width of the fixed stimulus with a center frequency of $3200 \mathrm{~Hz}$ should be approximately 0, but it can be seen from Fig. 2 that in fact it has a mean value of 0.043 . It is likely that this discrepancy was caused by a floor effect in the experiment due to the finite range of variation available with the variable stimulus. This may mean that the stimuli that were judged to be narrowest (those centered on 
1600 and $3200 \mathrm{~Hz}$ ) were actually narrower than the subjective results shown in Fig. 2 suggest and may also be the cause of the offset of 0.07 in the prediction model shown in Eq. (5). However, the likely error is small compared to the range of results for the other experimental stimuli, and therefore has not been corrected in the model.

It must be noted that the shape of the subjective result curve in Fig. 2 and the modeled result in Eq. (5) inevitably are partly influenced by the center frequencies of the stimuli that were used in the experiment, and that the use of a greater number of fixed stimuli with center frequencies between those used would allow for a more accurate model. Also, Eq. (5) has been derived to match the subjective results to IACC measurement results, and it may be necessary to revise this once further adaptations are made to the IACC calculation in later sections of the paper.

\section{E. Discussion}

The physiological explanation for these subjective results is as yet unknown; however, a mechanism for incorporating the results of this experiment into a model of auditory source width perception needs to be derived. Since the stimuli used in this experiment all have a 1-IACC of 0 , simply adding the baseline function shown in Eq. (5) to the measured 1-IACC should provide a realistic measure of perceived auditory source width for these stimuli. The validity of this approach when applied to the measured results of stimuli with a range of IACC values is tested implicitly in the experiments described below.

\section{EXPERIMENT 2-EFFECT OF BREAKDOWN OF PHASE LOCKING ON PERCEPTION OF IACC}

\section{A. Introduction}

As mentioned in the Introduction, it is expected that the results of the research that are reported in this paper will be implemented as part of a more complete model of binaural hearing. One feature that is commonly found in such models is a simulation of the breakdown of phase locking in the neural output of the ear above a certain frequency (Stern and Trahiotis, 1997).

Detailed models have been developed to mimic the limited firing rate of the neural synapses (Meddis et al., 1990), though this effect is commonly approximated by the use of half-wave rectification followed by a low-pass filter (e.g., Karjalainen, 1996). Bernstein and Trahiotis (1996b) investigated the effect of a half-wave rectification and low-pass filtering preprocess on IACC-based calculations used to predict the binaural detection performance of a group of subjects for a range of stimuli. They found that the addition of this preprocess led to predictions which matched their empirical results quite accurately. As binaural detection and perception of auditory source width appear to be similar in their association with the IACC, it is likely that this type of simulation of the breakdown of phase locking will be applicable to prediction of the perceived auditory source width of stimuli.

However, the majority of auditory source width models that are based on the IACC do not employ such a simulation.
In view of this, it is of interest to investigate whether phase locking has any effect on auditory source width perception and, if so, whether simulation of this using rectification and filtering is an appropriate addition to an auditory source width model. If this is the case, then the optimum filter characteristics must also be determined.

\section{B. Stimuli}

The amplitude-modulation-based stimuli that were used for the previous experiment have an interesting property that can be employed to investigate the effect of the breakdown of phase locking on the perceived auditory source width of a stimulus. If a stimulus with an IACC of 0 is created [therefore $G=0.5$ in Eqs. (3) and (4)] when $f_{c}=(2 n-1) f_{m}$ where $n$ is an integer, then each output channel contains only one tonal component, the frequency of which is $f_{c}+f_{m}$ in one channel and $f_{c}-f_{m}$ in the other. This means that there is no in-channel amplitude modulation, and the decorrelation of the two channels is due to the constantly varying phase difference between them caused by the different frequency tones in each channel. Conversely, when $G=0.5$ and $f_{c}$ $=2 n f_{m}$, the two tonal components are combined in each of the output channels, which causes in-channel amplitude modulation in each of them. In this case the decorrelation is caused by the fluctuations in the interchannel level difference over time as well as a change in the interchannel phase difference every quarter of the modulation period. This can be seen in the plots of the examples of each stimulus type shown in Fig. 3. For ease of reference, stimuli with $f_{c}$ $=(2 n-1) f_{m}$ will be referred to as type 1 , and stimuli with $f_{c}=2 n f_{m}$ will be referred to as type 2 .

For both of these types of stimuli, the IACC is 0. However, the IACC of the amplitude envelopes of the two signals is very different, where type 1 stimuli have an envelope IACC of 1, and type 2 stimuli have an envelope IACC that is much lower (the actual value of the IACC will be dependent on the method used to extract the envelope of the signal). This is shown in detail by Mason et al. (2003). While the envelope differences between type 1 and type 2 signals may not be perceived at lower frequencies, it is possible that they will be perceived at higher frequencies where the breakdown of phase locking may cause the human perceptual system to fail to track the fine detail of the stimuli.

For this experiment, the stimuli were created using the same suppressed carrier amplitude modulation technique that was used in the previous experiment, though in this case all the fixed stimuli used $G=0.5$ which resulted in an IACC of 0 . The center frequencies $\left(f_{c}\right)$ of the fixed stimuli were chosen to encompass the range of frequencies around which previous research indicated the breakdown of phase locking may start to take effect. Ten center frequencies (values of $f_{c}$ ) were used for each stimulus type-type 1: 180, 300, 420, $580,740,940,1180,1460,1820$, and $2260 \mathrm{~Hz}$; type 2: 200, 320, 440, 600, 760, 960, 1200, 1480, 1840, and $2280 \mathrm{~Hz}$. This gave 20 fixed stimuli for the experiment.

In order to compare like with like, two variable stimuli were used, one of each type. The frequencies of the variable stimuli were chosen to be well below the frequency at which the breakdown of phase locking may start to have an effect, 

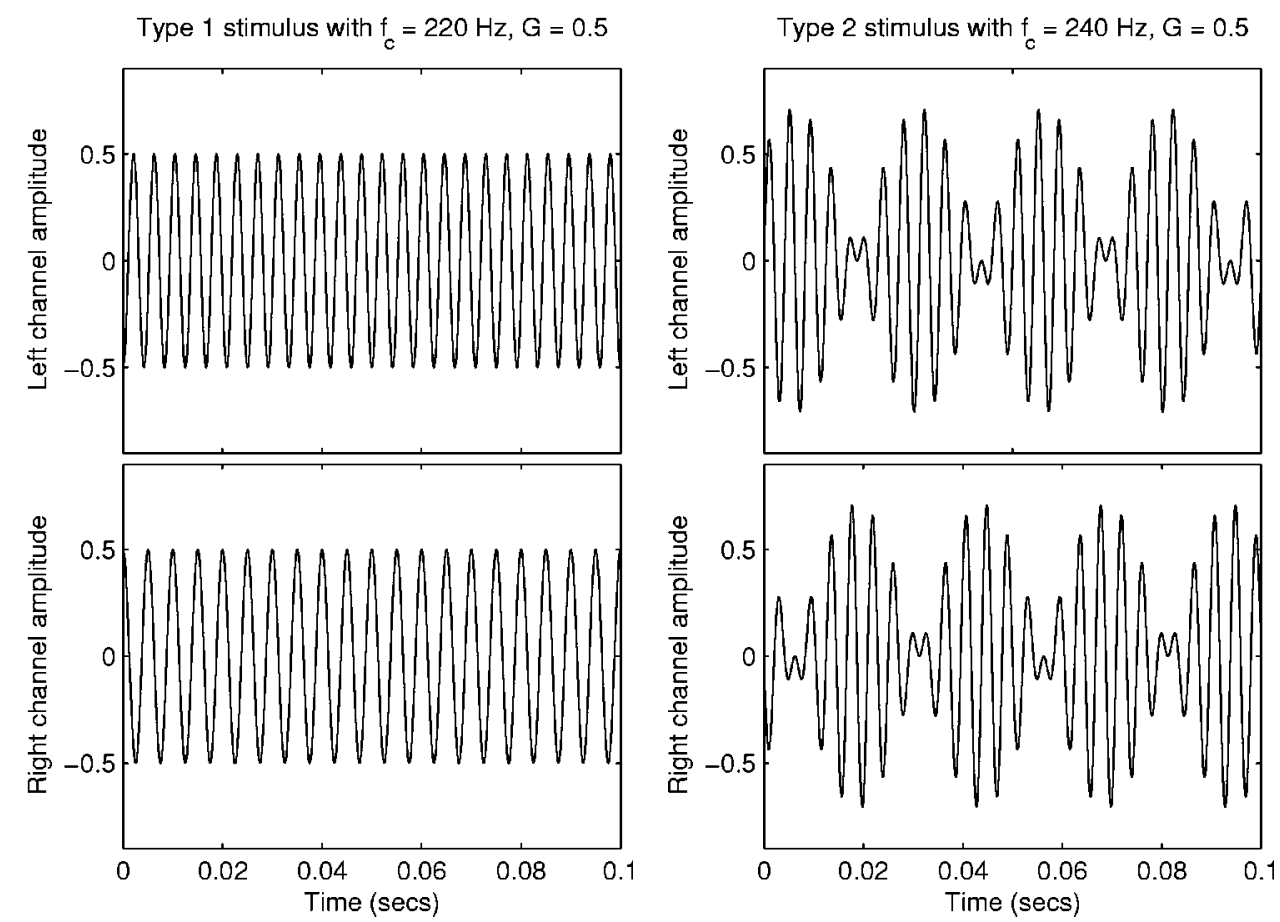

FIG. 3. An example signal of type 1 with a center frequency of $220 \mathrm{~Hz}$ and a gain $(G)$ of 0.5 in the left plot, and an example signal of type 2 with a center frequency of $240 \mathrm{~Hz}$ and a gain $(G)$ of 0.5 in the right plot. and to be able to cover as much of the range of auditory source widths of the fixed stimuli as possible, as judged by the authors in informal listening. The type 1 variable stimulus had a value of $f_{c}$ of $220 \mathrm{~Hz}$ and the type 2 variable stimulus had a value of $f_{c}$ of $240 \mathrm{~Hz}$. As for the previous experiment, the value of $f_{m}$ was fixed for all the stimuli at 20 $\mathrm{Hz}$.

\section{Procedure}

There were 20 fixed stimuli in the experiment; for each of these the subjects were asked to adjust the corresponding variable stimulus until they judged it to have the same auditory source width as the fixed stimulus. The experiment comprised two repetitions, each presented in a different random order. All seven selected subjects undertook the full experiment.

\section{Results}

The results of this subjective experiment were analyzed by calculating the IACC of the variable stimulus that the subjects judged to have the same perceived auditory source width as each fixed stimulus. The means and associated $95 \%$ confidence intervals of these results are shown in Fig. 4, with the center frequency of each fixed stimulus along the $x$ axis and 1-IACC of the matched variable stimulus on the $y$ axis, with the left plot showing the results for the type 1 stimuli and the right plot showing the results for the type 2 stimuli. In both plots, an increase on the $y$ axis can be interpreted as an increase in the perceived auditory source width.

As for the previous experiment, it is reasonable to expect that the subjects would adjust the IACC of the variable stimulus to be similar to the IACC of the fixed stimulus with a similar center frequency, i.e., in this case that the mean 1-IACC of the variable stimuli adjusted to match the width of the fixed stimuli with center frequencies of 180 and 200
$\mathrm{Hz}$ would be approximately 1. It can be seen in Fig. 4 that the variable stimuli matched to the width of the fixed stimuli centered on 180 and $200 \mathrm{~Hz}$ have IACCs of 0.89 and 0.8 , respectively. It is likely that the discrepancy between the expected and actual results is partially due to a ceiling effect in the experiment due to the finite range of variation available with the variable stimulus. The fact that there appears to be a greater difference between the expected and actual results for the type 2 stimuli than for the type 1 stimuli is explained later.

If the breakdown of phase locking in the ear does need to be taken into account in an IACC-based model of auditory source width, then this will be evident as differences between the results at similar frequencies for the two types of stimuli, especially at higher frequencies. It can be seen in Fig. 4 that the results for the type 1 stimuli above approximately $1 \mathrm{kHz}$ indicate that these were perceived to be significantly narrower than the type 2 stimuli with similar center frequencies. This indicates that the breakdown of phase locking does indeed affect the relationship between the IACC and perceived auditory source width, and that this should therefore be taken into account in a model.

The most suitable type of low-pass filter was then identified as follows. Each fixed stimulus used in the experiment was passed through a trial filter and its IACC was calculated to give the modeled result. The variable stimulus that each subject matched to it was analyzed similarly, and the means and associated $95 \%$ confidence intervals were calculated to give the subjective result. The results from the two sets of filtered IACC measurements were compared, with an appropriate offset to compensate for any outstanding frequencydependent differences between the variable and fixed stimuli. This procedure was repeated using a number of different trial filter types, including that proposed by Bernstein and Trahiotis (1996b). The best fit between the subjective and modeled results was found with the use of half-wave rectification 

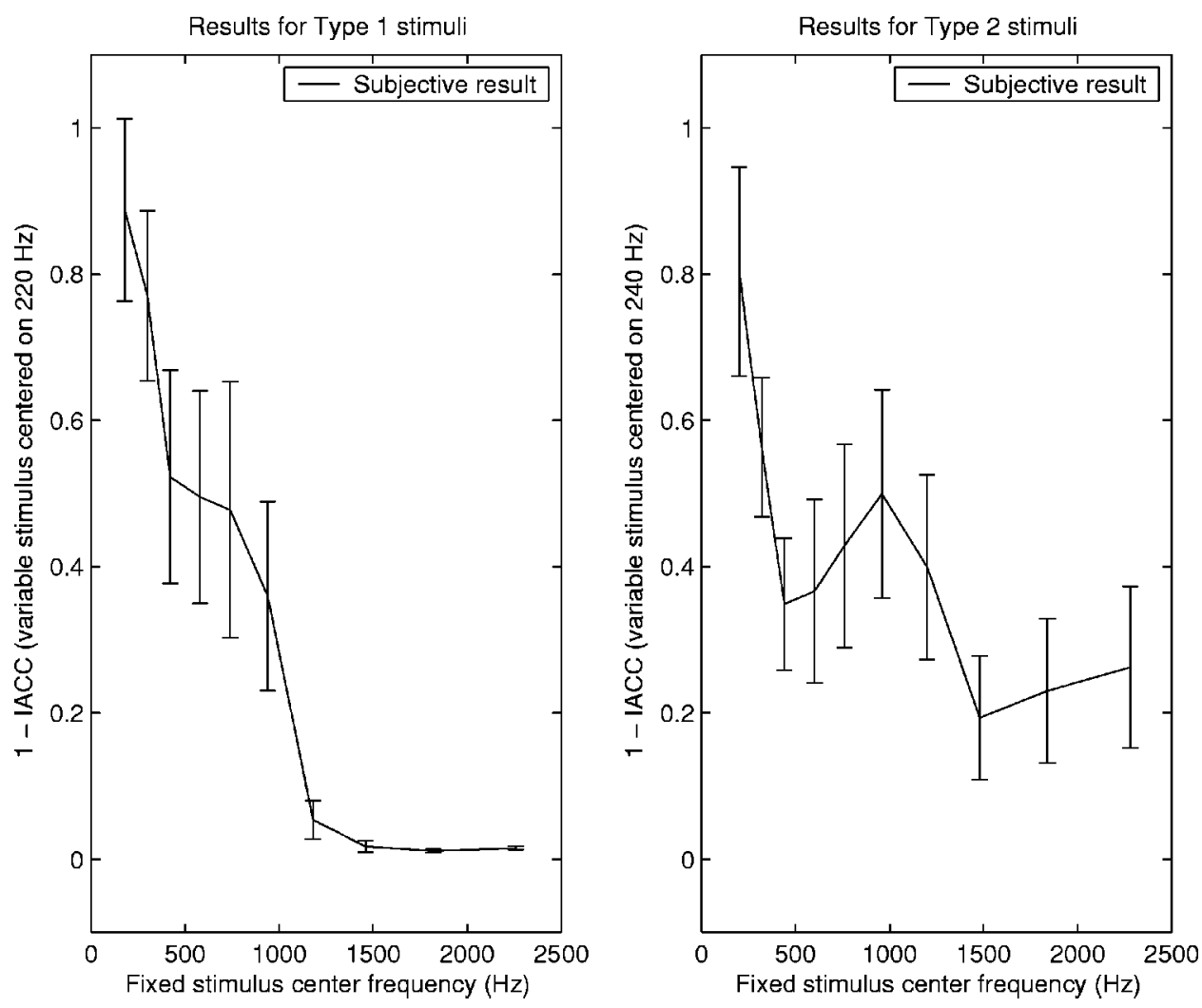

FIG. 4. Means and associated 95\% confidence intervals of the 1-IACC of the variable stimulus that the subjects judged to have the same auditory source width as each fixed stimulus in experiment 2 , with type 1 stimuli (variable stimulus centered on $220 \mathrm{~Hz}$ ) on the left and type 2 stimuli (variable stimulus centered on $240 \mathrm{~Hz}$ ) on the right.
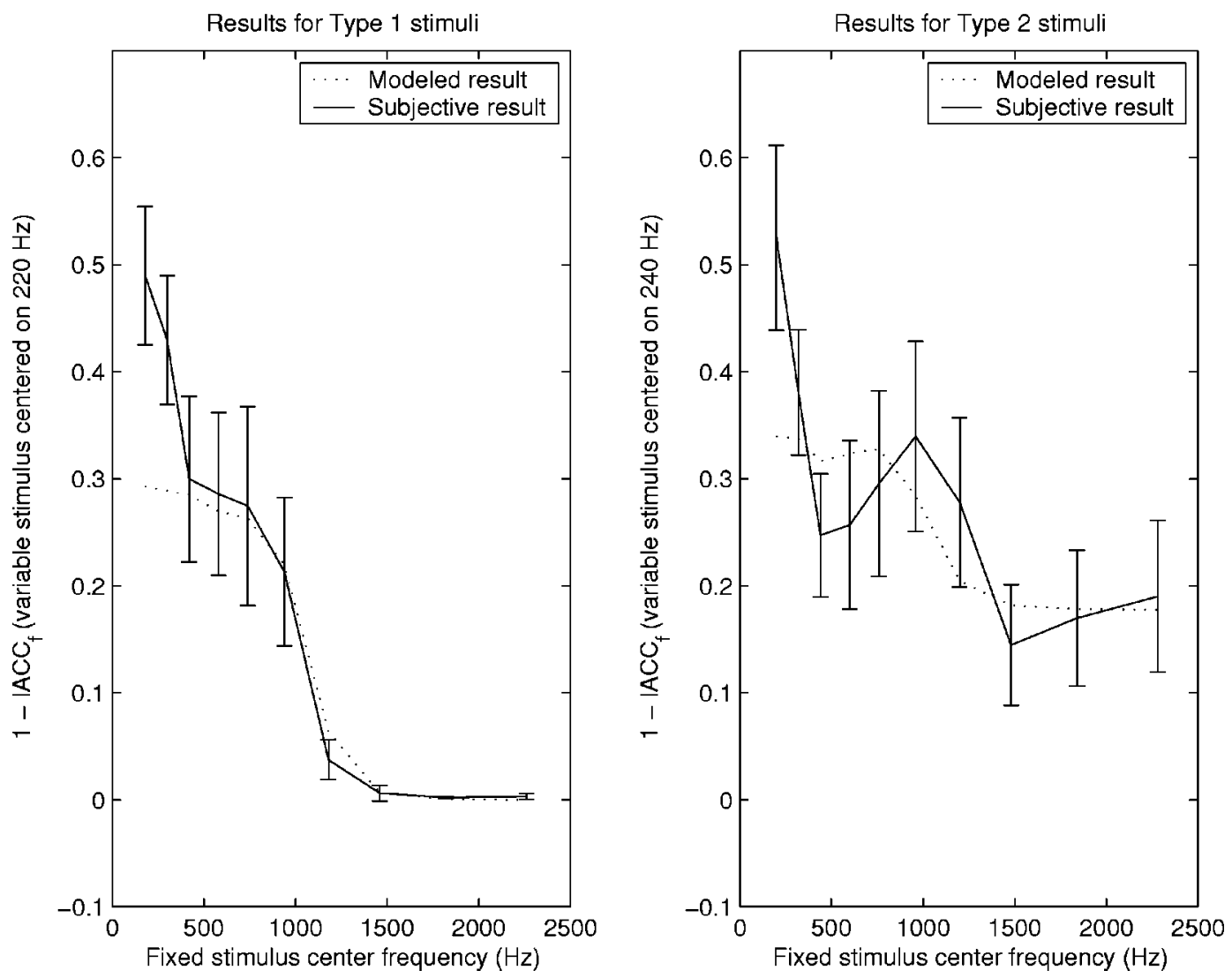

FIG. 5. Means and associated $95 \%$ confidence intervals of the 1-IACC $\mathrm{I}_{\mathrm{f}}$ of the variable stimulus that the subjects judged to have the same auditory source width as each fixed stimulus in experiment 2 (subjective result), together with the 1- $\mathrm{IACC}_{\mathrm{f}}$ of each fixed stimulus with the addition of an offset (modeled result), with type 1 stimuli (variable stimulus centered on $220 \mathrm{~Hz}$ ) on the left and type 2 stimuli (variable stimulus centered on $240 \mathrm{~Hz}$ ) on the right. 
followed by a sixth-order Butterworth low-pass filter with a cutoff frequency of $1 \mathrm{kHz}$. The relevant comparison is shown in Fig. 5 (the term 1-IACC $\mathrm{f}$ refers to a calculation made after half-wave rectification and filtering as described in the Appendix).

It can be seen in Fig. 5 that in terms of the shape of the subjective results in the region of $1 \mathrm{kHz}$ there is a difference between the two stimulus types. The authors currently cannot offer an explanation for this, and the optimum filter type was chosen based on matching the range of the $95 \%$ confidence intervals for as many data points as possible. It is also apparent from Fig. 5 that the modeled results do not accurately match the subjective results at low frequencies; it was therefore necessary to ignore the results at low frequencies in order to obtain a good fit. This mismatch at low frequencies may be caused by a further frequency dependency in the relationship between the IACC and the perceived auditory source width. However, further investigation is required to verify this.

\section{E. Discussion}

It must be noted that half-wave rectification of a signal causes the possible range of IACC values to reduce. An audio signal that has an IACC of 0 before half-wave rectification will have an IACC of approximately 0.4 after half-wave rectification. In a similar manner, an audio signal that has an IACC of -1 before half-wave rectification will have an IACC of approximately 0 after half-wave rectification. This difference in the measured IACC values is partly caused by the fact that half-wave rectified signals have a nonzero mean (i.e., they contain a dc offset). The effect that this has on the measured result could be negated by subtracting the mean value of each of the input signals before calculation, resulting in a cross-covariance calculation as defined by Lynn and Fuerst (1998). However, Bernstein and Trahiotis (1996a) showed that the cross-correlation calculation (that they termed the normalized correlation) gave a better match to the perceived properties of high-frequency signals than the cross-covariance calculation (that they termed the normalized covariance). For the purposes of the model of perceived auditory source width that is under development in this paper, using the cross-covariance calculation would result in an overestimation of the dissimilarity of the high-frequency signals that have been half-wave rectified and low-pass filtered.

It is possible that the use of a more physiologically accurate model of the transduction process of the ear will give an improved match to the subjective data, compared to halfwave rectification and low-pass filtering. However, this will be at the expense of an increase in the required computation, and the improvement in the match to the subjective data is likely to be small.

In view of this reduction in the range of possible IACC values, the function that was derived at the end of the previous experiment needs to be modified. In order to update this previous model, the analysis of that experiment was repeated using the 1-IACC $\mathrm{f}_{\mathrm{f}}$ calculation (including the half-wave rectification and low-pass filtering). This analysis resulted in a similar equation to that shown in Eq. (5). The result, now in terms of $1-\mathrm{IACC}_{\mathrm{f}}$, is shown in Eq. (6), where $b$ predicts the
$1-\mathrm{IACC}_{\mathrm{f}}$ of the variable stimulus whose auditory source width would be judged to match that of a fixed stimulus with center frequency $f$ :

$$
\begin{aligned}
& b=\left[\frac{\sin [(5.25 \sqrt{f}+4000) / 100]+0.75}{f^{0.2}}+0.05\right] / 3.8, \\
& 100 \leqslant f \leqslant 12800 .
\end{aligned}
$$

\section{F. Comparison between type 1 and type 2 variable stimuli}

In order to allow comparison between the results for the two types of stimuli, an additional experiment was conducted. Fixed type 2 stimuli were created with a center frequency $\left(f_{c}\right)$ of $240 \mathrm{~Hz}$ and IACC values of $0.0,0.2,0.4,0.6$, 0.8 , and 1.0. The subjects were then asked to match the perceived auditory source width of these stimuli using a type 1 variable stimulus with a center frequency $\left(f_{c}\right)$ of $220 \mathrm{~Hz}$. The same seven selected subjects undertook this experiment, and they were asked to adjust the variable stimulus until they judged it to have the same auditory source width as each fixed stimulus in turn, as for the previous experiments. The experiment comprised two repetitions, each with the fixed stimuli presented in a different random order.

The results of this experiment were analyzed by calculating the IACC of the variable stimulus that the subjects judged to have the same perceived auditory source width as each fixed stimulus. The means and associated 95\% confidence intervals of these results are shown in Fig. 6, with the 1-IACC of each fixed stimulus (type 2 centered on $240 \mathrm{~Hz}$ ) along the $x$ axis and the 1-IACC of the matched variable stimulus (type 1 centered on $220 \mathrm{~Hz}$ ) on the $y$ axis.

These results indicate that for the variable type 1 stimulus to have the same auditory source width as the fixed type 2 stimulus with a given 1-IACC value, the former requires a lower 1-IACC value than the latter. In addition, it appears that the perceived auditory source width of the fixed type 2 stimuli with 1-IACC values of 0.8 and 1 are both matched by the same type 1 stimulus. These results can be interpreted as follows. First, it appears that the type 1 stimulus with a given 1-IACC value will be perceived to be slightly wider than the type 2 stimulus with the same 1-IACC value. Second, it appears that the auditory source width of the type 2 stimulus does not increase if the 1-IACC is increased from approximately 0.8 to 1 . It is likely that the latter effect is the cause of the larger discrepancy between the expected and actual results for the type 2 stimulus at $200 \mathrm{~Hz}$ compared to the type 1 stimulus at $180 \mathrm{~Hz}$. While the discrepancy between the IACCs is greater, the fact that there appears to be little change in the perceived width between a 1-IACC of 0.8 and a 1-IACC of 1 for the associated variable stimulus may mean that the perceptual discrepancy is small. For the stimuli that were tested, a reasonable conversion from the 1-IACC results for the type 2 stimuli to the 1-IACC results for the type 1 stimuli can be made by multiplying the former by 0.85 . 


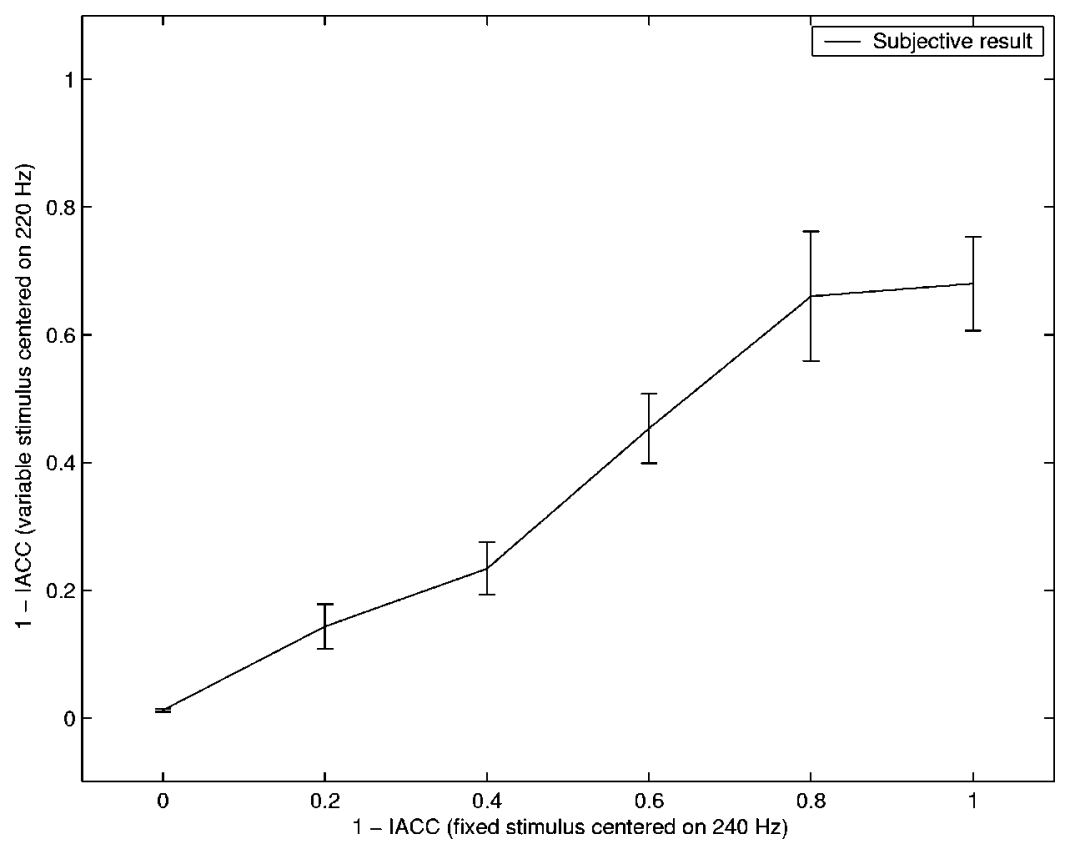

FIG. 6. Means and associated $95 \%$ confidence intervals of the 1-IACC of the type 1 variable stimulus with a center frequency of $220 \mathrm{~Hz}$ that the subjects judged to have the same auditory source width as the type 2 fixed stimuli with a center frequency of $240 \mathrm{~Hz}$ and a range of 1-IACC values.

\section{EXPERIMENT 3-INVESTIGATION OF LOW-FREQUENCY STIMULI WITH IACC $=0$}

\section{A. Introduction}

For some time, the relationship between the IACC and the perceived auditory source width at low frequencies has been considered to be a problem. For instance, signals that contain spectral information solely below approximately 500 $\mathrm{Hz}$ generally exhibit little variance in IACC compared to signals that contain spectral information solely above this range, and at low frequencies natural sounds rarely have an IACC much less than 1 (Hidaka et al., 1995). This is thought to be due to the small physical distance between the ears compared to the wavelength of the sound for low-frequency stimuli, though this is complicated by the diffraction and scattering of the sound by the head (Lindevald and Benade, 1986). Tohyama and Suzuki (1989) found that the modeling equation derived by Cook et al. (1955) could be used to predict the IACC of a diffuse field, but only by using an equivalent ear spacing of $32.8 \mathrm{~cm}$ as opposed to the true interaural distance of approximately $16 \mathrm{~cm}$.

Although the IACC of low-frequency stimuli (below a few hundred Hertz) rarely varies far from a value of 1, research has shown that low-frequency stimuli can vary significantly in their perceived auditory source width (Morimoto and Maekawa, 1988; Griesinger, 1997; Martens, 1999). Griesinger (1992) suggests that it is possible that the human auditory system compensates for the lack of decorrelation at low frequencies. The disparity between lowfrequency IACC and perceived auditory source width has led previous investigations into IACC-based models of perceived auditory source width to recommend ignoring the results at low frequencies (Hidaka et al., 1995). However, it is important to deal with the disparity in order to create a model that can accurately predict the perceived auditory source width of a wide range of stimuli.

In view of the above, an experiment was conducted to investigate whether the relationship between the IACC and the perceived auditory source width is dependent on frequency at low frequencies, and, if so, to derive an appropriate model.

\section{B. Stimuli}

The amplitude-modulation-based stimuli described in Eqs. (1)-(4) were again used for this experiment. All of the fixed stimuli had a value of $G$ of 0.5 , giving an IACC of 0 . Fourteen different center frequencies (values of $f_{c}$ ) were used for each type of stimulus-type 1: 100, 140, 180, 260, $300,340,380,420,460,580,660,740,820$, and $940 \mathrm{~Hz}$; type $2: 120,160,200,280,320,360,400,440,480,600$, $680,760,840$, and $960 \mathrm{~Hz}$. In order to reduce the number of judgments required in the experiment, a number of the fixed stimuli that were identical to those in the previous experiment were omitted from this experiment, and for these the results were taken from the previous experiment. However, four of the fixed stimuli were identical between the two experiments in order to test the repeatability of the results and to confirm that the results from the two experiments could be combined.

As for the previous experiment, two types of variable stimuli were employed in order that the experiment compared like with like with regard to stimulus type. The same variable stimuli were used as in the previous experiment (type $1 f_{c}=220 \mathrm{~Hz}$ and type $2 f_{c}=240 \mathrm{~Hz}$ ), in order to be able to combine the results from the two experiments. However, it is possible that the maximum perceivable auditory source width available with these variable stimuli is not as great as the auditory source width of the lower frequency fixed stimuli, which may cause a ceiling effect in the results. This will be considered in the analysis and discussion of the results. 

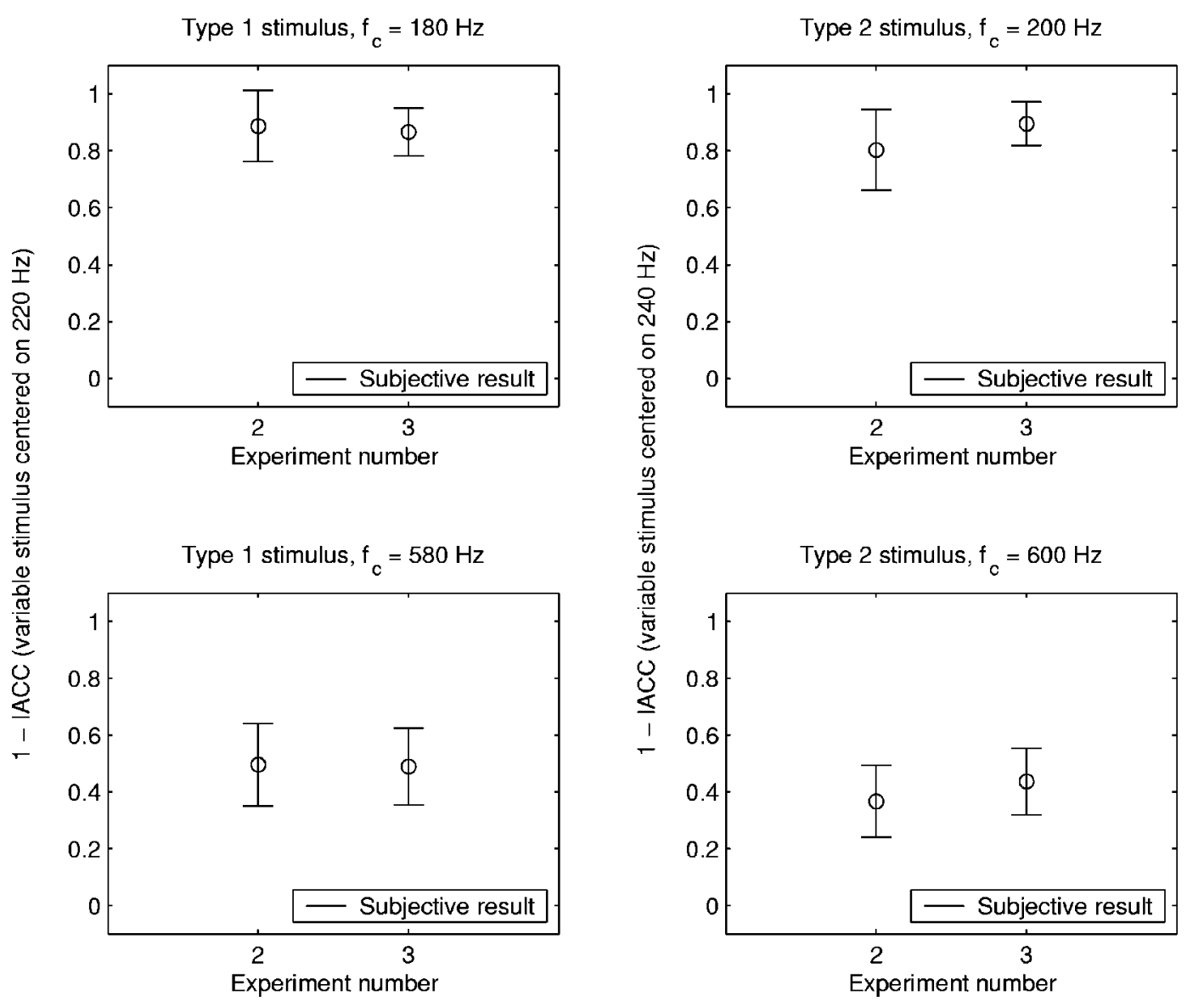

FIG. 7. Means and associated 95\% confidence intervals of the 1-IACC of the variable stimulus that the subjects judged to have the same auditory source width as each fixed stimulus that was identical in experiments 2 and 3, separated by the stimulus type and the fixed stimulus center frequency.

\section{Procedure}

There were 20 fixed stimuli in the experiment; for each of these the subjects were asked to adjust the corresponding variable stimulus until they judged it to have the same auditory source width as the fixed stimulus. The experiment comprised two repetitions, each presented in a different random order. All seven selected subjects undertook the full experiment.

\section{Results}

The first stage of the analysis involved an examination of the similarity between the results of this experiment and the results of the previous experiment for the four repeated fixed stimuli, in order to determine whether the results from the two experiments could be combined. As for the previous experiments, the analysis involved calculating the IACC of the variable stimulus that the subjects judged to have the same perceived auditory source width as each fixed stimulus. The means and associated 95\% confidence intervals of these results, shown in Fig. 7, display no statistically significant differences between the two experiments, which means that the data from the two experiments can be combined.

The means and associated $95 \%$ confidence intervals of the combined results are shown in Fig. 8, with the measured 1-IACC of the variable stimulus that the subjects considered to be the same auditory source width as each fixed stimulus on the $y$ axis, and the center frequency of the fixed stimulus on the $x$ axis, separated by the stimulus type.

The results shown in Fig. 8 support those from the previous experiment, in that the perceived auditory source width of a stimulus appears to increase as its center frequency falls below approximately $400 \mathrm{~Hz}$. Above $400 \mathrm{~Hz}$, the perceived auditory source width appears to be almost constant up to the frequency at which the breakdown of phase locking starts to take effect. It must be noted that the perceived auditory source width of the stimuli at low frequencies may be greater than that indicated in Fig. 8, as it is possible that the results below approximately $200 \mathrm{~Hz}$ were subject to a ceiling effect caused by the limited maximum auditory source width of the variable stimulus.

When investigating a suitable method for modeling this frequency dependency, it was logical to start with the research of Lindevald and Benade (1986) and Tohyama and Suzuki (1989). As the results of these two studies were almost identical over the frequency range tested, the simpler formula of Tohyama and Suzuki was employed, the equivalent of which is shown in Eq. (7), where $s$ is the mean IACC in a diffuse field, $f$ is the frequency, $d$ is equivalent to the distance between the ears (calculated by Tohyama and Suzuki to be $0.328 \mathrm{~m}$ ), and $c$ is the speed of sound in air:

$$
s=\operatorname{sinc}(2 f d / c) .
$$

The accuracy of the match between the experiment results 

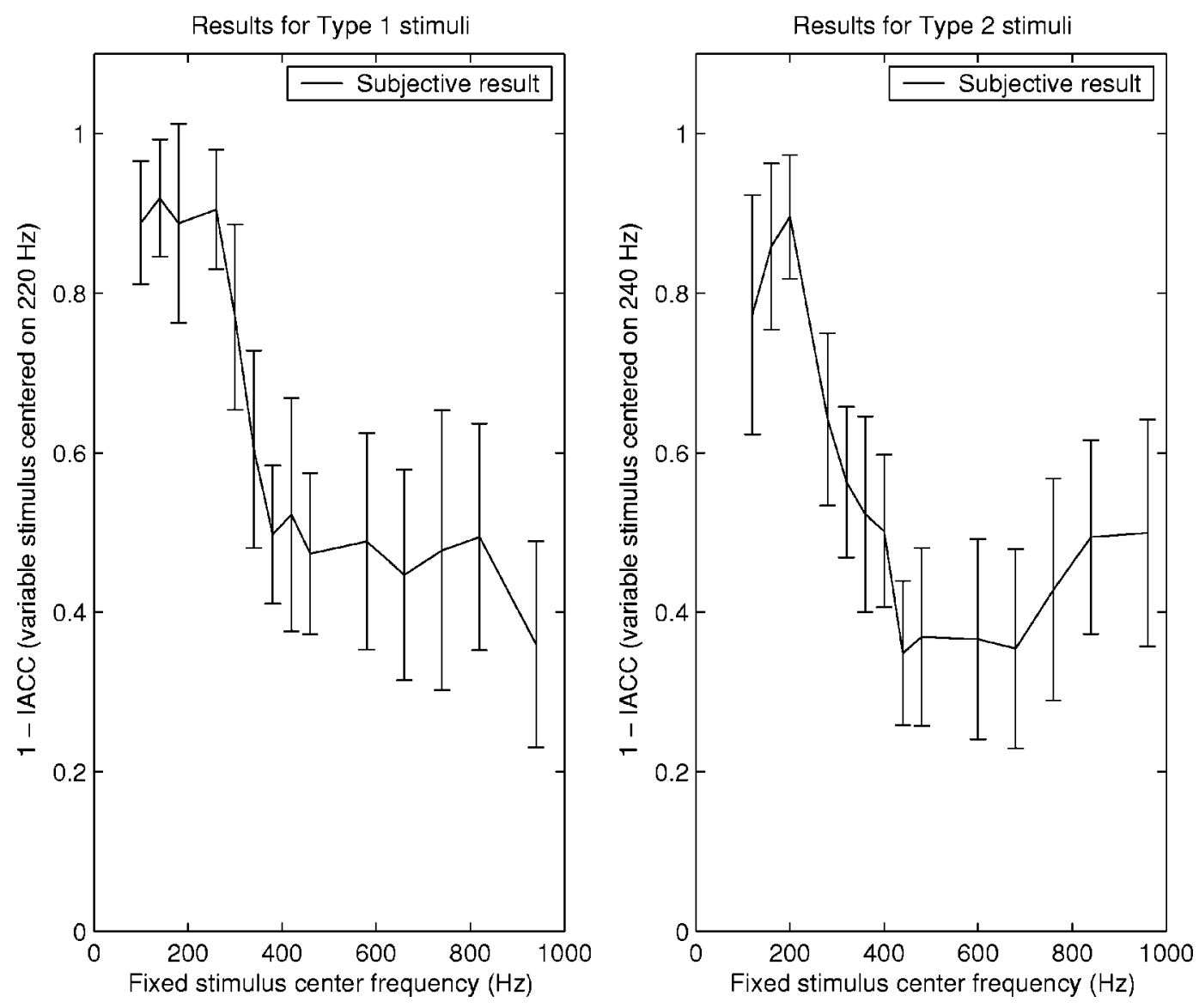

FIG. 8. Means and associated 95\% confidence intervals of the 1-IACC of the variable stimulus that the subjects judged to have the same auditory source width as each fixed stimulus in experiment 3, with type 1 stimuli (variable stimulus centered on $220 \mathrm{~Hz}$ ) on the left and type 2 stimuli (variable stimulus centered on $240 \mathrm{~Hz}$ ) on the right.

and the predictions from this formula was evaluated as follows. An IACC was calculated for each of the fixed stimuli after preprocessing with the half-wave rectification and lowpass filtering derived from experiment 2 (giving a result in terms of $1-\mathrm{IACC}_{\mathrm{f}}$ ). This value was then modified by multiplying it by $s$ [Eq. (7)] (giving a result in terms of $1-\mathrm{IACC}_{\mathrm{fs}}$ ). Finally, in order to examine the effect of the baseline compensation derived from experiment $1, b$ [Eq. (6)] was added (giving a result in terms of $1-\mathrm{IACC}_{\mathrm{fsb}}$ ). This is termed the modeled result. The variable stimulus that the subjects matched to each fixed stimulus was also processed using this method (giving a result in terms of $1-\mathrm{IACC}_{\mathrm{fsb}}$ ), and the means and associated $95 \%$ confidence intervals were calculated to give what is termed the subjective result.

The use of a frequency-dependent multiplier based on $s$, calculated using values of $d=0.328 \mathrm{~m}$ and $c=330 \mathrm{~ms}^{-1}$, improved the match between the subjective and modeled results. However, it was found that using values of $d=0.4 \mathrm{~m}$ and $c=330 \mathrm{~ms}^{-1}$ in Eq. (8) resulted in a closer match. The results of this are shown in Fig. 9:

$$
s=\max (\operatorname{sinc}(2 f d / c), 0) / 3+0.5 .
$$

It is apparent from the results shown in Fig. 9 that the modifications made to the basic IACC calculation [half-wave rectification and low-pass filtering of the input signal, multiplication of the result by the low-frequency compensation $s$ of Eq. (8), and addition of the baseline compensation $b$ of Eq.
(6)] mean that the modeled results closely match the subjective results judgments in most cases. However, it is also apparent that the modeled results for the low-frequency stimuli (below approximately $200 \mathrm{~Hz}$ ) are higher than the subjective results. As noted above, it is possible that the limited maximum perceived auditory source width of the variable stimulus caused the auditory source width of these low-frequency fixed stimuli to be underestimated. In view of this, it is possible that the matching at low frequencies is actually better than these results suggest.

\section{E. Discussion}

It is unclear why this experiment shows a better match between the modeled results and subjective results for a value of $d$ in Eq. (8) of 0.4 compared to the value of 0.328 that matched the results of modeled measurements in a diffuse field in previous studies. If it is assumed that the frequency dependency at low frequencies is due to the human perceptual system compensating for the limited range of IACC values encountered at those frequencies, then it is possible that the difference between the actual interaural distance and the interaural distance that gives the most accurate prediction of the subjective results is due to overcompensation by the auditory system. However, it is not possible, from the current results, to accurately determine the cause of this effect. 

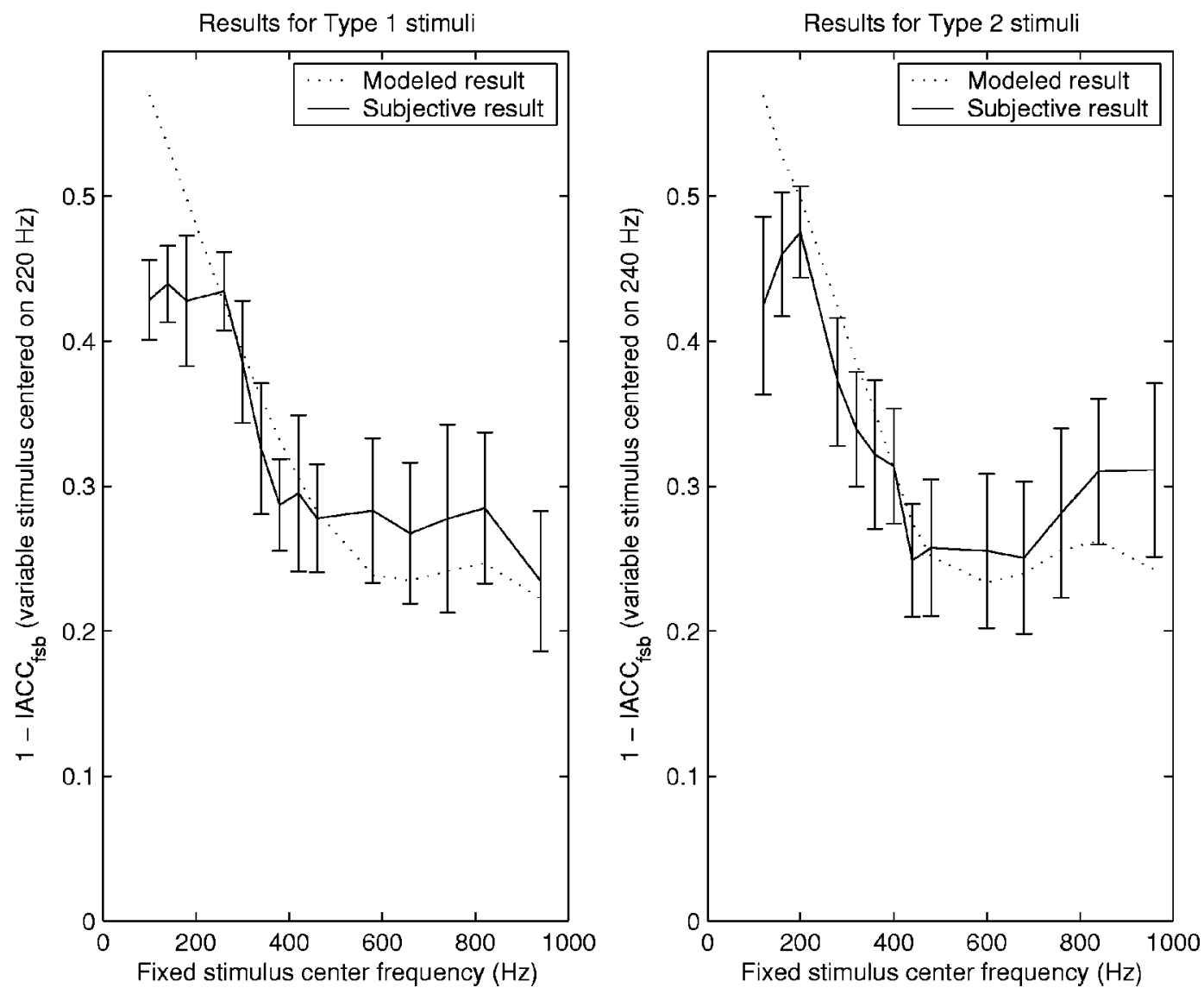

FIG. 9. Means and associated $95 \%$ confidence intervals of the 1 - IACC $_{\mathrm{fsb}}$ of the variable stimulus that the subjects judged to have the same auditory source width as each fixed stimulus in experiment 3 (subjective result), together with the 1 -IACC $\mathrm{fsb}_{\mathrm{fb}}$ of each fixed stimulus (modeled result), with type 1 stimuli (variable stimulus centered on $220 \mathrm{~Hz}$ ) on the left and type 2 stimuli (variable stimulus centered on $240 \mathrm{~Hz}$ ) on the right.

\section{CONCLUSIONS}

Three experiments were conducted to investigate features of the frequency dependency of the relationship between the IACC of an auditory stimulus and its perceived auditory source width. Three important findings emerged.

First, the perceived auditory source width of a stimulus with an IACC of 1 decreases monotonically as its frequency increases from a hundred to a few thousand Hertz, beyond which the perceived auditory source width increases again. The effect on the perceived auditory source width has been modeled with a simple formula, whose application in the subsequent experiments indicates that it provides a reasonable match to the subjective results.

Second, for IACC-based models of perceived auditory source width, it is important to take into account the loss of fine temporal detail at higher audio frequencies that results from the breakdown of phase locking in the neural output of the ear. This loss of detail causes the perceived auditory source width of higher frequency stimuli to be dependent on the IACC of the envelope of the signal, rather than the signal itself. This effect can be modeled by passing the input signal through a half-wave rectifier and a sixth-order low-pass Butterworth filter with a cutoff frequency of $1 \mathrm{kHz}$ prior to measuring the IACC of the signal.

Finally, for low-frequency stimuli, human auditory perception appears to make up for the limited range of IACC values encountered in real situations by introducing an addi- tional frequency dependency in the relationship between the IACC and the perceived auditory source width at these frequencies. This can be modeled by multiplying the IACC by a frequency-dependent factor derived from the expected range of IACC values that may be encountered in natural sound fields.

These three findings can be combined into a single generic model termed 1-IACC $\mathrm{fsb}_{\mathrm{fb}}$, which is described by Eq. (9), where $w$ is a prediction of the perceived auditory source width, $l_{f}$ and $r_{f}$ are the left and right binaural signals that have been half-wave rectified and filtered with a sixth-order Butterworth low-pass filter with a cutoff frequency of $1 \mathrm{kHz}$, $s$ is the frequency-dependent weighting factor defined in Eq. (8), and $b$ is the frequency-dependent baseline function defined in Eq. (6):

$w=\left[1-\max _{-1}\left(\frac{\int_{t 1}^{t 2} l_{f}(t) r_{f}(t+\tau) d t}{\left[\int_{t 1}^{t 2} l_{f}^{2}(t) d t \int_{t 1}^{t 2} r_{f}^{2}(t) d t\right]^{1 / 2}}\right)\right] s+b$.

\section{DISCUSSION}

The experiments that are described in this paper used stimuli whose characteristics were tightly controlled and constant over time. These were employed so that it was possible to match an objective value to a subjective effect as accurately as possible. However, the disadvantage of using 
tightly controlled stimuli is that they are less externally valid (i.e., they are dissimilar to natural audio signals).

The stimuli had a narrow bandwidth and were time invariant, both in terms of their spectral content and their IACC. The perceived auditory source width of stimuli whose characteristics vary over time has not yet been determined. Additionally, the optimum method for combining measurements that are made in a number of separate frequency bands has not yet been derived. These factors must be investigated in order to further develop the model such that it can reasonably be used to predict perceived auditory source width for naturally occurring audio signals.

This paper only considered stimuli that have positive IACC values, as it has been shown that signals with a negative IACC are perceived to have differing spatial properties compared to equivalent signals with a positive IACC (Plenge, 1972). Further research is required into the perceived effect of stimuli with a negative IACC to allow this factor to be modeled.

The model that has been derived from the results of these experiments gives a prediction of the perceived auditory source width of the stimuli on a scale that allows comparison between stimuli, but is not related to any physical measurement of width. It would be useful to be able to predict the perceived auditory source width of stimuli in terms of a physical distance across for sounds perceived to be within the head, or in terms of a subtended angle for sounds perceived to be outside the head. Further research is required to convert the results of the measurement model to such a scale.

\section{ACKNOWLEDGMENTS}

This work was supported by the Engineering and Physical Sciences Research Council (EPSRC), UK, Grant No. GR/ R55528/01. The authors would like to thank Slawomir Zielinski and Søren Bech for their comments on earlier versions of this paper. The authors would also like to thank Armin Kohlrausch and two anonymous reviewers for their helpful comments.

\section{APPENDIX: CALCULATION DETAILS}

A number of different types of IACC-based calculation have been used in this paper. This appendix explains these in detail.

The basis for each calculation is the normalized crosscorrelation function (NCC). In order for this to be equivalent to the IACC, it is essential that the signals analyzed are identical to those at the ears of the listener. The NCC is shown in Eq. (A1), where $x$ and $y$ are the two signals whose correlation is to be calculated, $t 1$ to $t 2$ is the period over which the correlation is calculated, and $\tau$ is an offset between the two signals under measurement:

$$
\operatorname{NCC}(\tau)=\frac{\int_{t 1}^{t 2} x(t) y(t+\tau) d t}{\left[\int_{t 1}^{t 2} x^{2}(t) d t \int_{t 1}^{t 2} y^{2}(t) d t\right]^{1 / 2}} .
$$

The NCC is calculated across a range of $\tau$ that is equivalent to $\pm 1 \mathrm{~ms}$, and the IACC is taken to be the maximum value across this range. The values of $t 1$ and $t 2$ are set to give a window length of $50 \mathrm{~ms}$, and measurements are made at 1.5 -ms steps throughout the input signal to quantify any variations in IACC over time. However, only the mean of these results is ultimately used. For the stimuli that were used in the experiment, it was found that the variations in IACC over time measured in this manner were no more than \pm 0.01 . The input signal is not filtered or weighted unless otherwise stated.

The variations on this basic calculation were derived from the results of the experiments and are denoted using subscript characters. A list of these is given below.

IACC - basic IACC calculation.

1-IACC-basic IACC calculation with the result then subtracted from 1.

$1-\mathrm{IACC}_{\mathrm{f}}$-the input signal is half-wave rectified and low-pass filtered using a sixth-order Butterworth filter with a cutoff frequency of $1 \mathrm{kHz}$ before using the basic IACC calculation with the result then subtracted from 1.

$1-\mathrm{IACC}_{\mathrm{fs}}$-the input signal is half-wave rectified and low-pass filtered using a sixth-order Butterworth filter with a cutoff frequency of $1 \mathrm{kHz}$ before using the basic IACC calculation with the result then subtracted from 1 and multiplied by the frequency-dependent weighting shown in Eq. (8).

$1-\mathrm{IACC}_{\mathrm{fsb}}$ - the input signal is half-wave rectified and low-pass filtered using a sixth-order Butterworth filter with a cutoff frequency of $1 \mathrm{kHz}$ before using the basic IACC calculation with the result subtracted from 1, multiplied by the frequency-dependent weighting shown in Eq. (8), and then added to the frequency-dependent baseline function shown in Eq. (6).

All of the measurements described in the paper were made by analyzing the two-channel experimental stimuli in the digital domain. In order to ensure that the characteristics of the stimuli that the subjects heard were similar to those measured in the digital domain, a number of stimuli were reproduced over the system used in the experiment and captured using a head and torso simulator (HATS). It was found that the measurements made in the digital domain were within \pm 0.02 of those measurements made of the signals that were captured with the HATS.

Ando, Y. (1985). Concert Hall Acoustics (Springer-Verlag, Berlin).

Bernstein, L. R., and Trahiotis, C. (1992). "Discrimination of interaural envelope correlation and its relation to binaural unmasking at high frequencies," J. Acoust. Soc. Am. 91, 306-316.

Bernstein, L. R., and Trahiotis, C. (1996a). "On the use of the normalized correlation as an index of interaural envelope correlation," J. Acoust. Soc. Am. 100, 1754-1763.

Bernstein, L. R., and Trahiotis, C. (1996b). "The normalized correlation: Accounting for binaural detection across center frequency," J. Acoust. Soc. Am. 100, 3774-3784.

Blauert, J., and Lindemann, W. (1986). "Spatial mapping of intracranial auditory events for various degrees of interaural coherence," J. Acoust. Soc. Am. 79, 806-813.

Boehnke, S. E., Hall, S. E., and Marquardt, T. (2002). "Detection of static and dynamic changes in interaural correlation," J. Acoust. Soc. Am. 112, 1617-1626.

Brand, A., Behrend, O., Marquardt, T., McAlpine, D., and Grothe, B. (2002). "Precise inhibition is essential for microsecond interaural time difference coding," Nature (London) 417, 543-547.

Cardozo, B. L. (1965). "Adjusting the method of adjustment: SD vs DL," J. Acoust. Soc. Am. 37, 786-792.

Chernyak, R. I., and Dubrovsky, N. A. (1968). "Pattern of the noise images and the binaural summation of loudness for the different interaural corre- 
lation of noise," Proc., 6th Int. Con. Acoust., A53-A56.

Constan, Z. A., and Hartmann, W. M. (2001). "Sound localization by interaural time differences at high frequencies," J. Acoust. Soc. Am. 109, 2485.

Cook, R. K., Waterhouse, R. V., Berendt, R. D., Edelman, S., and Thompson, M. C. (1955). "Measurement of correlation coefficients in reverberant sound fields," J. Acoust. Soc. Am. 27, 1072-1077.

Culling, J. F., and Summerfield, Q. (1998). "Measurements of the binaural temporal window using a detection task," J. Acoust. Soc. Am. 103, 35403553.

de Vries, D., Hulsebos, E., and Baan, J. (2001). "Spatial fluctuation in measures for spaciousness," J. Acoust. Soc. Am. 110, 947-954.

Gabriel, K. J., and Colburn, H. S. (1981). "Interaural correlation discrimination: I. Bandwidth and level dependence," J. Acoust. Soc. Am. 69, $1394-1401$.

Gescheider, G. A. (1997). Psychophysics, the Fundamentals (Erlbaum, Hillsdale, NJ).

Glasberg, B. R., and Moore, B. C. J. (1990). "Derivation of auditory filter shapes from notched-noise data," Hear. Res. 47, 103-138.

Grantham, D. W., and Wightman, F. L. (1979). "Detectability of a pulsed tone in the presence of a masker with time-varying interaural correlation," J. Acoust. Soc. Am. 65, 1509-1517.

Griesinger, D. (1992). "IALF-Binaural measures of spatial impression and running reverberance," Aud. Eng. Soc. Preprints, Vol. 92, No. 3292.

Griesinger, D. (1997). "The psychoacoustics of apparent source width, spaciousness and envelopment in performance spaces," Acustica 83, 721731.

Hesse, A. (1986). "Comparison of several psychophysical procedures with respect to threshold estimates, reproducibility and efficiency," Acustica 59, 263-273.

Hidaka, T., Beranek, L. L., and Okano, T. (1995). "Interaural crosscorrelation, lateral fraction, and low- and high-frequency sound levels as measures of acoustical quality in concert halls," J. Acoust. Soc. Am. 98, 988-1007.

ISO (1987). “Acoustics-Normal equal-loudness level contours," ISO 226.

ISO (1997). "Acoustics-Measurement of the reverberation time of rooms with reference to other acoustical parameters," ISO 3382.

Karjalainen, M. (1996). "A binaural auditory model for sound quality measurements and spatial hearing studies," Proc., Int. Conf. Acoust. Sp. Sig. Proc. (ICASSP), pp. 985-988.

Keet, W. de V. (1968). "The influence of early lateral reflections on the spatial impression," Proc. 6th Int. Con. Acoust., pp. E53-E56.

Kurozumi, K., and Ohgushi, K. (1983). "The relationship between the cross-correlation coefficient of two-channel acoustic signals and sound image quality," J. Acoust. Soc. Am. 74, 1726-1733.

Levitt, H. (1971). "Transformed up-down methods in psychoacoustics," J. Acoust. Soc. Am. 49, 467-477.

Lindevald, I. M., and Benade, A. H. (1986). "Two-ear correlation in the statistical sound fields of rooms," J. Acoust. Soc. Am. 80, 661-664.

Lynn, P. A., and Fuerst, W. (1998). Introductory Digital Signal Processing with Computer Applications (Wiley, Chichester).
Martens, W. L. (1999). "The impact of decorrelated low-frequency reproduction on auditory spatial imagery: Are two subwoofers better than one?" Proc., 16th Int. Aud. Eng. Soc. Conf., pp. 67-77.

Mason, R., Brookes, T., and Rumsey, F. (2003). "Creation and verification of a controlled experimental stimulus for investigating selected perceived spatial attributes," Aud. Eng. Soc. Preprints, Vol. 114, No. 5771.

Meddis, R., Hewitt, M. J., and Shackleton, T. M. (1990). "Implementation details of a computational model of the inner hair-cell/auditory-nerve synapse," J. Acoust. Soc. Am. 87, 1813-1816.

Morimoto, M., and Iida, K. (1995). "A practical evaluation method of auditory source width in concert halls," J. Acoust. Soc. Jpn. (E) 16, 59-69.

Morimoto, M., and Maekawa, Z. (1988). "Effects of low frequency components on auditory spaciousness," Acustica 66, 190-196.

Morimoto, M., Iida, K., and Furue, Y. (1993). "Relation between auditory source width in various sound fields and degree of interaural crosscorrelation," Appl. Acoust. 38, 291-301.

Morimoto, M., Ueda, K., and Kiyama, M. (1995). "Effects of frequency characteristics of the degree of interaural cross-correlation and sound pressure level on the auditory source width," Acustica 81, 20-25.

Okano, T. (2000). "Image shift caused by strong lateral reflections, and its relation to inter-aural cross correlation,” J. Acoust. Soc. Am. 108, 22192230

Perrott, D. R., Musicant, A., and Schwethelm, B. (1980). "The expandingimage effect: The concept of tonal volume revisited,' J. Aud Res. 20, 43-55.

Plenge, G. (1972). "Über das problem der im-kopf-lokalisation (On the problem of in-head localization)," Acustica 26, 241-252.

Robinson, D. E., and Jeffress, L. A. (1963). "Effect of varying the interaural noise correlation on the detectability of tonal signals," J. Acoust. Soc. Am. 35, 1947-1952.

Sakai, S., Asahi, N., Gotoh, T., Yoshii, F., and Akiya, H. (1980). "On the simplified measurement of the interaural crosscorrelation coefficient and its effects on sound imaging and quality," Aud. Eng. Soc. Preprints, Vol. 67, No. 1697.

Sayers, B. M. (1964). "Acoustic-image lateralization judgments with binaural tones," J. Acoust. Soc. Am. 36, 923-926.

Sayers, B. M., and Cherry, E. C. (1957). "Mechanism of binaural fusion in the hearing of speech," J. Acoust. Soc. Am. 29, 973-987.

Stern, R. M., and Trahiotis, C. (1997). "Models of binaural perception," in Binaural and Spatial Hearing in Real and Virtual Environments, edited by R. H. Gilkey and T. R. Anderson (Erlbaum, Hillsdale, NJ), Chap. 24, pp. 499-531.

Tohyama, M., and Suzuki, A. (1989). "Interaural cross-correlation coefficients in stereo-reproduced sound fields," J. Acoust. Soc. Am. 85, 780786.

Wier, C. C., Jesteadt, W., and Green, D. M. (1976). "A comparison of method-of-adjustment and forced-choice procedures in frequency discrimination," Percept. Psychophys. 19, 75-79.

Yanagawa, H., Anazawa, T., and Itow, T. (1990). "Interaural correlation coefficient and their relation to the perception of subjective diffuseness," Acustica 71, 230-232. 